\title{
Research of Converged Cable Access Platform in Tri-networks Integration
}

\author{
Yepeng Ni \\ Computer and Network Center \\ Communication University of China \\ Beijing, 100024, China \\ e-mail:nyp_2010@cuc.edu.cn
}

\author{
Cheng Yang, Jianbo Liu \\ Information Engineering School \\ Communication University of China \\ Beijing, 100024, China \\ e-mail:1jb@cuc.edu.cn
}

\begin{abstract}
In an age of tri-networks integration, CATV operators are facing huge competitive pressure from telecommunication operators. The "transformation" is the only way of CATV operators to cope with the competition. Such CATV operators should transform a single TV service provider to a multiple service operator (MSO) which includes video service, high speed internet service and multimedia voice service. This paper proposed a novel CATV system architecture which is called converged cable access platform (CCAP). The comprehensiveness of CCAP covers all the business of trinetworks integration which can be supported by the CATV network. And it is a good way for the development of the CATV network in the future. The shortage of trunk fiber resources is the problem in provisioning of CCAP. This paper introduced the WDM technology advantages, technical classification and the development situation, to explore how to achieve trunk fiber resource upgrade and to meet the growing demands of users on the basis of not change the existing optical network structure.
\end{abstract}

Keywords-Tri-networks Integration; CATV; CCAP; CWDM; user experiences

\section{INTRODUCTION}

The development of CATV linked to the development and progress of information technology. Today, thanks to the development of digitalization and IP technology, a new era of tri-networks integration has been opened.

In an age of tri-networks integration, CATV operators are facing huge competitive pressure from telecommunication operators [1]. On the one hand, the market share of telecom industry has occupied the absolute leading position, with the strongly advancing of broadband access on the basic of years of telephone communication business which depends on its years of policies and resource advantages. On the other hand, IPTV business is gaining momentum, participate in the market competition with integration business which will be bound with the voice and broadband access business.

The way of CATV operators to cope with competition is transformation that is to transform a single TV service provider to a MSO which includes video service, high speed internet service and multimedia voice service. This is not only the requirements of technical systems, but comprehensive requirements of market oriented, overall business and operational ability. But the most important thing is the customers' experience of high quality. The customers have the right in accordance with the quality of its services feel to choose service operators. As operators participated in market competition, they must win customers by the high quality customer service experience, besides, all activities that operators launch are centered on quality customer service experience.

Under the background of tri-networks integration, CATV operators should give customer following experiences:

(1)User can enjoy the service of broadcasting, narrowcasting, high speed internet access, multimedia audio, wireless broadband video at home, and implementation across the screen video services.

(2)There are different product portfolios of users' favorite well-known service brand, and more business bundling can be done.

(3) Convenient for users to open business (including one-stop customer service, user self-service, etc.).

(4) Ensuring high quality of business services, and handling user fault quickly.

(5) Unified user bills.

In order to achieve the user experience above, CATV operators should upgrade their headend from the traditional one to the CCAP network architecture. In section two, the paper will introduce the CCAP. In section three, the paper will discuss the problem of shortage of fiber resource, and propose WDM to cope with the problem. The conclusion is in the last section.

\section{CCAP TECHNICAL PRESENTATIONS}

The CCAP provide novel headend architecture for CATV operators and a new equipment architecture option for manufacturers.

In this section, we will discuss the background of CCAP, the CCAP network architecture, the CCAP operation features and its benefits.

\section{A. CCAP background overview}

With the rise of narrow-multicast and unicast services, service providers need more equipment such as CMTS, EQAM to service users, need flexible management of many businesses. For example[2], Beijing Gehua CATV network has promoted the high- 
definition interactive digital TV service after years of efforts, that high-definition interactive digital television users will reach 2.6 million units by the end of 2011 and it at the same time, actively explore the development of the broadband Internet access which is more than 100000 subscribers, besides, it actively carry out the experiments of IP voice services; Based on trinetworks integration, the following problems must be solved by Gehua to fully meet the high quality demand of the user experience:

(1)How to satisfy more applications of narrowcasting QAM for VOD and SDV?

(2)How to meet the IP access bandwidth requirements which can be comparable to those of competitors?

(3) How to meet the increasing equipment covers and power requirements in network front-end?

In order to solve the above problems, the CableLabs issues the CCAP technical report in 2011.

The Converged Cable Access Platform (CCAP) is intended to provide a new equipment architecture option for manufacturers to achieve the Edge QAM and CMTS densities that MSOs require in order to address the costs and environmental challenges resulting from the success of narrowcast services [3]. The CCAP leverages existing technologies, including DOCSIS 3.0, modular headend architecture (MHA), and current HFC architectures; and also can include newer ones, such as Ethernet optics and EPON (Ethernet Passive Optical Network).

\section{B. CCAP network architecture}

The initial discussion paper has two CCAP architecture, one is I-CCAP architecture, and the other is M-CCAP architecture, the M-CCAP architecture were removed in Cablelabs final release version, that is, retaining only the I-CCAP architecture. CCAP's original intention and goal is to simplify the space, in a platform solve all the problems, but M-CCAP put different functions into different racks, which is somewhat contrary to the CCAP original intention, so that later M-CCAP architecture were removed in the final release version, and also in this paper too.

I-CCAP architecture is shown in following Fig. 1.

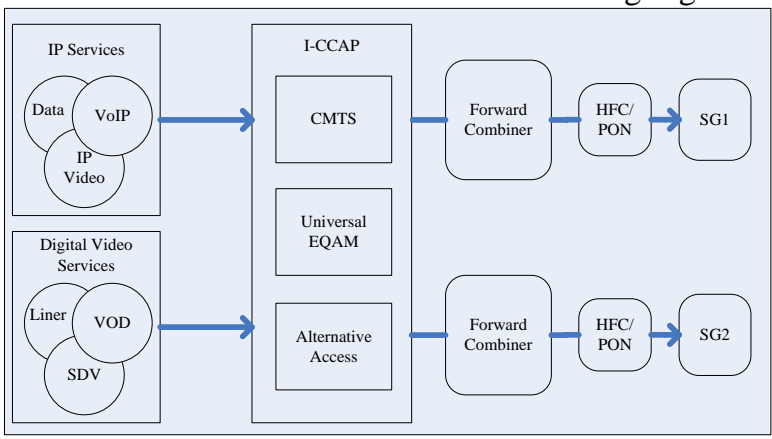

Figure 1. I-CCAP architecture
There are three parts in this figure. The left part indicate the two resource of CCAP. One is the IP service resources which include data service, VoIP service and IP video service. Another one is the digital video service resources which include liner video, VOD and SDV. The data is send from tow resource to ICCAP in different ways. The IP service resource use TCP to send IP packets and the digital video service resource use UDP to send MPEG TS data. The mid part is I-CCAP which contains three modules, the CMTS, the universal EQAM and the alternative access. The CMST is a piece of equipment, typically located in a cable company's headend or hub-site, which is used to provide high speed data services, such as cable Internet or VoIP, to cable subscribers. The universal EQAM is a device that allows MSOs a future direction for cost-competitive, all-digital migration and creates a common converged platform for an assortment of new services mostly based on video, data and voice. The alternative access is a module which provides compatibility client interfaces for CCAP. The last part depicts the user's network situation. From the above discussion, we can drawn that CCAP mainly to provide users with IP services and digital video services, it combine the CMTS and EQAM functions in one equipment and provide PON access option for high speed service.

CCAP headend acquire video from various sources to be provided to the subscriber via the access network. In Fig. 2, the dotted lines represent the video data while the remainder of the diagram represents control elements or flows within the MPEG video system.

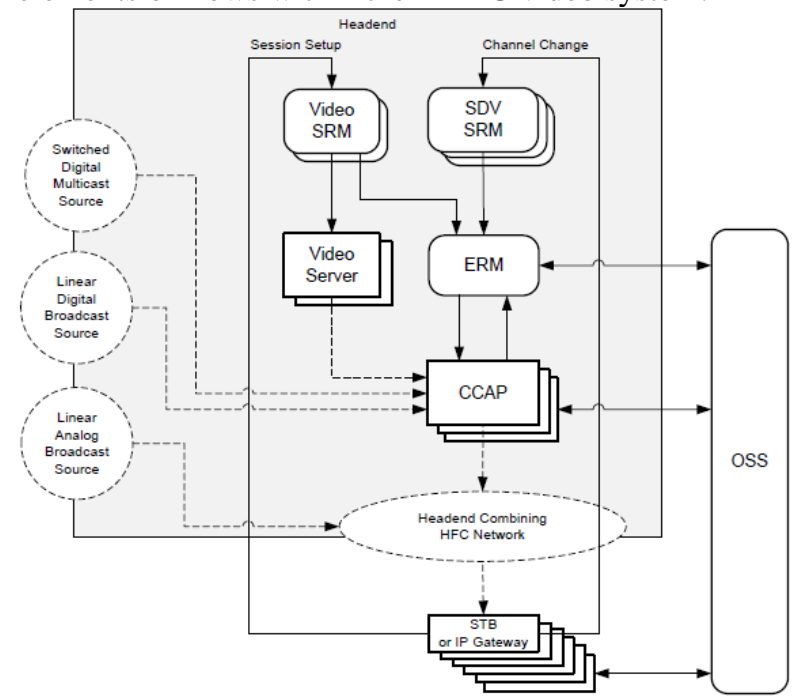

Figure 2. CCAP video headend reference architecture

Digital video that is not broadcast continuously to service groups is controlled by the interaction of a service-specific client application on the STB, signaling to service-specific session resource managers (SRM) to request receipt of a video stream. When the STB client 
requests a stream, the SRM must acquire the necessary resources that allow the stream to be transported from source to destination. The CCAP will be directed to route them to a particular QAM port and RF frequency, either via a UDP port mapping scheme, where the UDP port defines the RF port and frequency, or under the control of the Edge Resource Manager (ERM). In either case, the CCAP will be required to multiplex incoming SPTSs into MPTSs, and perform QAM modulation, upconversion, and in some cases content scrambling.

The CCAP performs all DOCSIS functions in the way that a traditional CMTS platform does. In addition, PON can be deployed on the CCAP to manage commercial HSI traffic. The following diagram, Fig. 3, illustrates how HSI streams flow through the CCAP and the network to DOCSIS and PON devices and back.

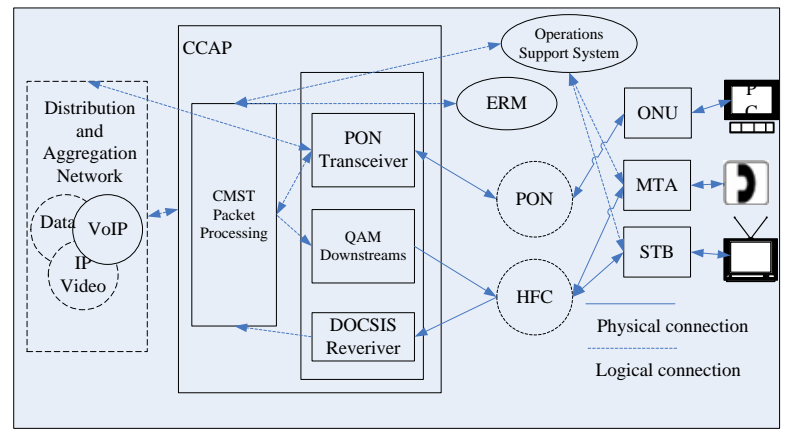

Figure 3. CCAP data reference architecture

The CCAP receives IP content from a distribution and aggregation network, via one or more physical interfaces. The CCAP performs all of the MAC-layer functionality and all the initialization and operational DOCSIS-related processes. The MAC-layer functionality includes all signaling functions, downstream bandwidth scheduling, and DOCSIS framing. The CCAP creates the DOCSIS QAMs for the service groups the CCAP serves, and these QAMs are modulated and output as either downstream QAMs on the downstream line cards in the CCAP, or QPSK on the downstream PON interfaces.

The outputs of the CCAP are combined with legacy OOB data (and possibly legacy analog video) in the headend combining network and are distributed through the HFC network or PON network, where they are received by the following devices:

(1)MTA: Provides PacketCable voice services and DOCSIS HSI for telephony.

(2)ONU: Terminates the PON traffic for business services applications.

(3)Set-top Boxes: native QAM video and command and control data (either through DOCSIS or legacy outof-band).

Upstream DOCSIS HSI and PacketCable voice traffic travel through the HFC or EPON and are received by either QAM upstream receivers or PON transceivers.

\section{CCAP operation features}

Compare with the traditional CMTS and EQAM, CCAP have some new features. In this section, we will discuss those important ones.

\section{1) QAM Replication}

In order to simplify integration of the CCAP into existing systems, the CCAP is expected to implement a QAM replication feature. The purpose of this feature is to allow an operator to create logical service groups on a decoupled, service-by-service basis. This will provide the ability to replicate narrowcast video (SDV and VOD) QAMs across multiple ports on a given line card.

To effectively deal with service group inequalities, the CCAP will share a given set of SDV or VOD QAMs to other ports on the line card to form service groups with unique sets of HSI QAMs.

This is illustrated in the Fig. 4; note that each DLC port has a unique HSI group of QAMs (not depicted).

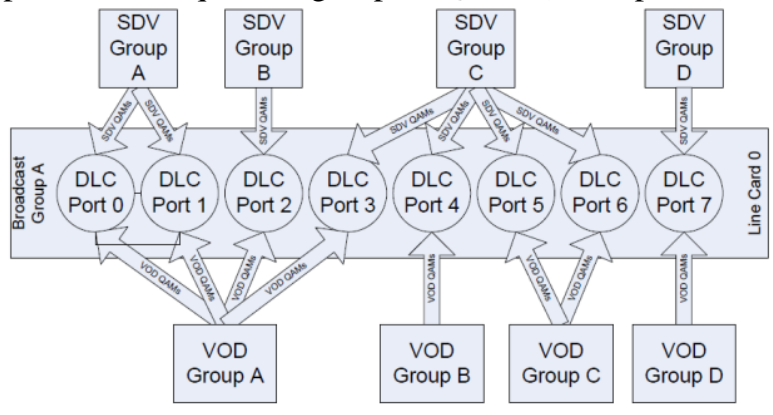

Figure 4. QAM Replication

\section{2) Optional Content Protection}

The CCAP provides an option to accept incoming transport streams that have had network encryption applied to keep them protected as they traverse the network of the cable operator, remove that network encryption, and then, based on the encryption mode specified for the content, apply the appropriate conditional access (CA) encryption for downstream transmission. The CCAP Decryptor and CCAP Scrambler are the two core optional functions of the CCAP, daisy-chained as shown in Fig. 5, to dynamically process a large portion of the input payload, according to the requirements of each content protection session.

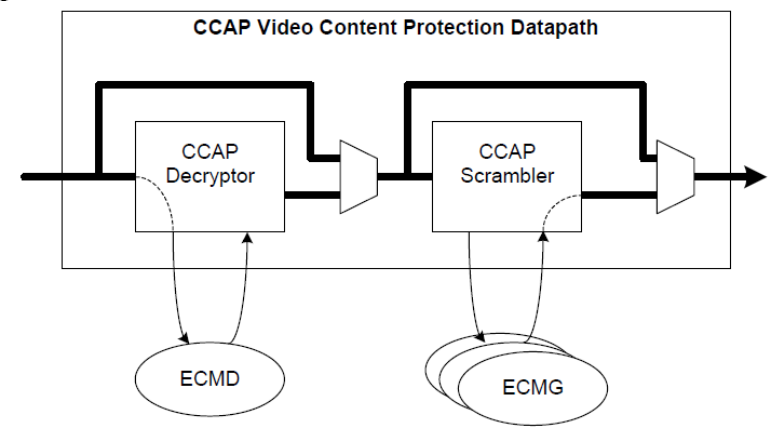

Figure 5. Optional CCAP Video Content Protection 
The CCAP content protection data path is designed to support all of the decryption and content protection needs for the VOD, SDV and linear digital broadcast.

3) CCAP Configuration Management

In order to provide operators the simplest path to deployment of CCAP with existing OSS systems, the goal for configuration and management of the CCAP is to treat the configuration of these very distinct platforms in a consolidated fashion.

The whole CCAP is complete equipment, so it only has one control point, and every object belong to it have only one configuration model. This changing greatly reduces configuration complexity.

\section{4) $\mathrm{DPoE}$}

The DOCSIS Provisioning of EPON (DPoE) specifications provide a service overlay of the DOCSIS and CMTS management framework on an IEEE 802.3ah/av EPON network. DPoE systems rely on the EPON MAC and PHY and the upper-layer DOCSIS protocols defined by CableLabs. The DOCSIS MAC and PHY do not apply.

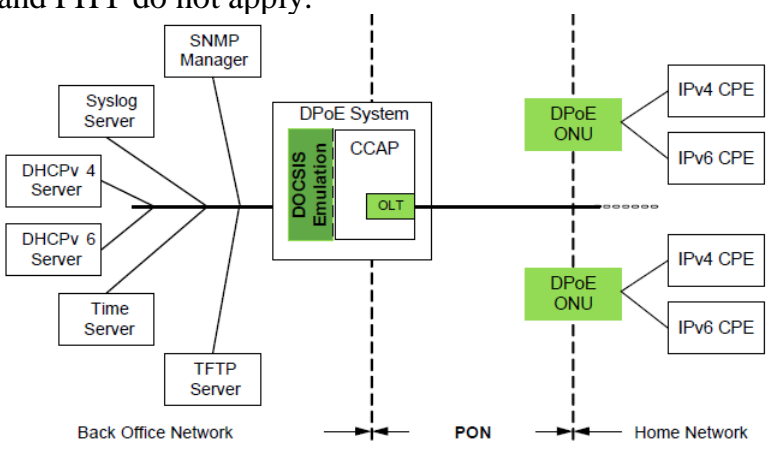

Figure 6. DPoE network architecture

Fig. 6 is shown the DPoE network architecture. The DPoE specifications define a system that is analogous to a CMTS. The DPoE System need not be a single device, but instead could be a collection of devices that includes an Optical Line Termination (OLT), router, and DOCSIS emulation system. Collectively, these separate devices would be referred to as the DPoE System. The DPoE System provides the logical interfaces and protocol translations necessary to integrate EPON devices into the DOCSIS OSSI framework. This permits operators to take advantage of standard EPON functionality while retaining their investment in back office operations and systems, leaving CPE unchanged.

\section{CCAP benefits}

There are many reasons for CCAP success amongst MSOs even before the equipment becomes available [4]. One of the key benefits of the CCAP is to achieve significant environmental efficiencies. To that end, Fig. 7 and Fig. 8 demonstrate an example of the space and power savings achieved by deployment of the CCAP in a typical system.
Fig. 7 depicts a typical installation in a headend consisting of the various digital services, including broadcast, SDV, VOD, and HSI equipment, plus the corresponding combiner and lasers/receivers.

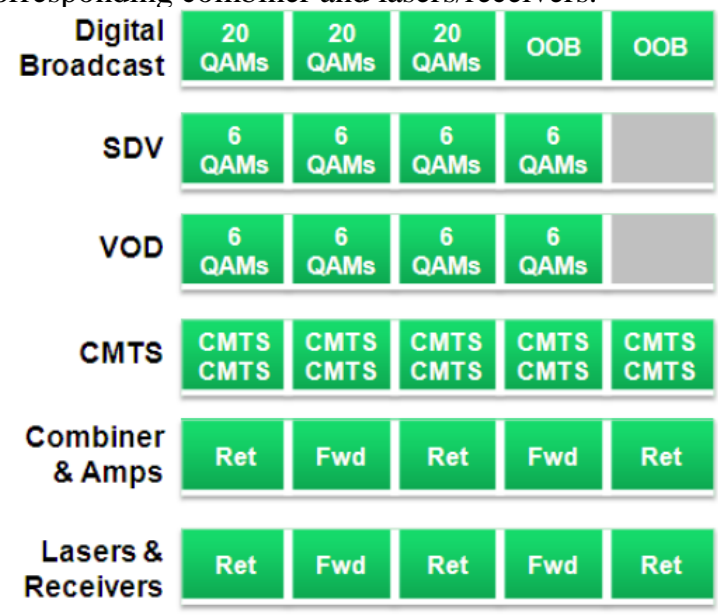

Figure 7. Typical Headend Space Usage

The example shown in Fig. 7 is intended to serve a typical population, combined in such a way as to result in 160 HSI service groups, and 120 VOD and matching SDV service groups.

Fig. 8 depicts the analogous installation when considering the deployment of equivalent CCAP equipment in a medium-sized chassis.

As shown in Fig. 8, about half of the space previously required is needed for deploying the CCAP. Moreover, given that the CCAP can serve twice as many narrowcast QAM channels as the previous architecture could, the depicted CCAP scenario actually results in even greater space savings, providing twice as much capacity in half the space.

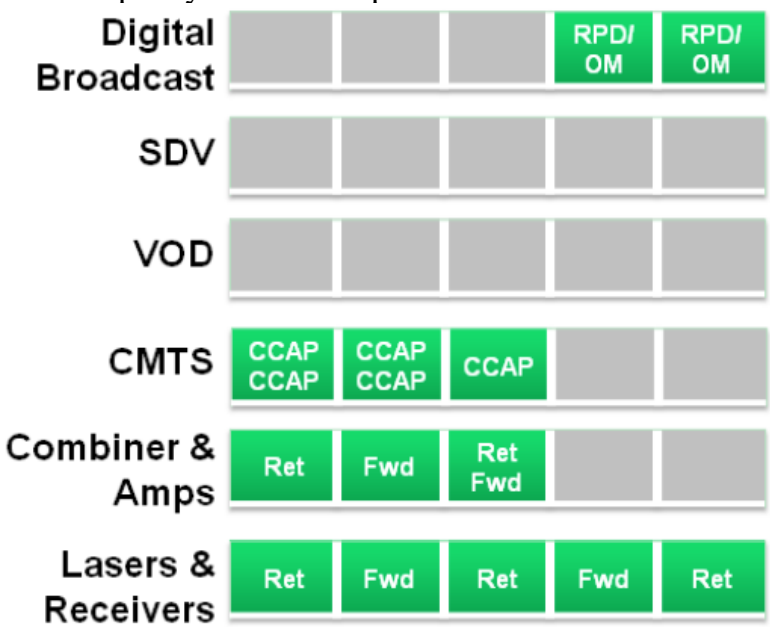

Figure 8. CCAP Deployment Space Usage

The other CCAP benefit which also exciting the MSOs are the RF Combining Simplifications. Deployment of the CCAP simplifies the RF combiner 
network by providing all QAM channels for all digital services from a single RF port, only leaving certain legacy functions for RF combining. Rather than having to rewire the physical plant to make service group changes, the QAM content of a downstream RF port can be changed via the CCAP configuration interface. Downstream of the CCAP, legacy out-of-band, analog channels, and maintenance streams (balance, sweep) are the only things that need to be combined into the CCAP output.

For all the above reasons, MSOs planning to take advantage of CCAP benefits as soon as the equipment becomes available might chose to address the operational changes that could be required as soon as practical [5].

\section{CWDM APPLICATION IN CCAP NETWORK}

In order to meet the need of optical access for replacing copper access of HFC network, reduce the service group coverage and expand the user access bandwidth, it isn't suitable to use the way of rebuilding access fiber trunk lines in large scale, and we must consider the upgrade a smooth, quick and feasible method. In author's opinion, we can adopt CWDM (Coarse where Division Multiplexing) technology to solve the problem of shortage of trunk fiber resources, which are shown in Fig. 9 and Fig. 10 [6].

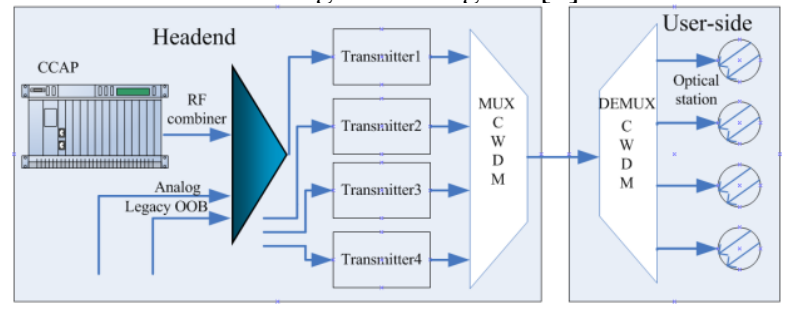

Figure 9. CWDM application in downstream system

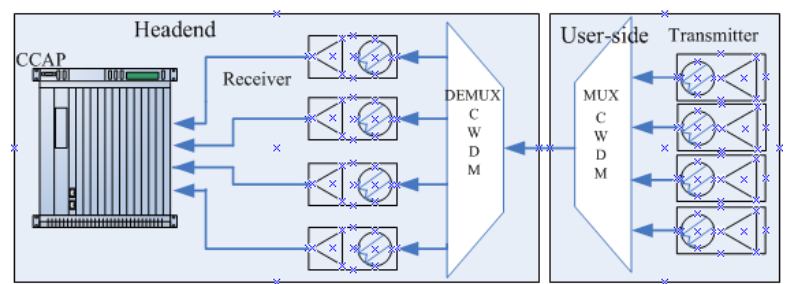

Figure 10. CWDM application in upstream system

This fiber optic trunk network access scheme is better to support the comprehensive utilization of radio, narrowcasting and data business and the IP application of future CATV network business, and the development of user access bandwidth requirements. The author thinks that it is an economic and feasible scheme. External CWDM device can maintain the original optical transmission equipment to use, but it is better that the transmission equipment manufacturer built in CWDM device to simplify the complexity of the equipment installation and commissioning. It is also needed that the cable network operators to do to do good cut in the wavelength of the deployment planning and allocation plan, for the network maintenance and management.

\section{CONCLUSION}

CCAP is the latest and important cable technology mechanism emerging in both American and European markets. In this article, the background and architectures of CCAP are introduced, a variety of configuration mode, system interfaces, network deployment and reliability mechanism of CCAP are described, and the CWDM application in CCAP network is discussion here. CCAP can achieve effective conversion of traditional video transmission to the way of the next generation of video transmission; it has an important significance on IP-based video and network DVR business. In an age of tri-networks integration, we can foresee the CCAP play a huge role for the development of MSOs.

\section{ACKNOWLEDGEMENTS}

This work is supported by the research program of The State Administration of Radio Film and Television (No.2012-27), by the program for National Science \& Technology Pillar Plan (No. 2012BAH02B03) and by the Natural Science Fund by The Communication University of China (No. 31322014XNG1364).

\section{REFERENCES}

[1] Wen Jianwei, Wang Houqin. Evaluation and enlightenment of international tri-networks integration. TV Engineering, 2010, 34(6) : 113-115.

[2] Liu zelin. Research of Beijing CATV development strategy in Tri-networks integration. Southwest Jiaotong University, 2010.

[3] Cablelabs. CCAP Architecture Technical Report CM-TRCCAP-V02-110614, 2011

[4] Jorge Salinger, Ty Pearman. CCAP: More than just box replacement.

http://www.cedmagazine.com/articles/2012/04/ccap-morethan-just-box-replacement, 2012.

[5] Jorge Salinger, Ty Pearman. Deploying CCAP: Not just another box swap, http://www.cedmagazine.com/articles/2013/06/deploying-ccapnot-just-another-box-swap, 2013.

[6] Banerjee A, Park Y, Clarke F, et al. Wavelength-divisionmultiplexed passive optical network (WDM-PON) technologies for broadband access: a review. Journal of optical $\begin{array}{llr}\text { networking, } & \text { 2005, } & 4(11):\end{array}$ 